\title{
Hyperuricemia as a possible risk factor for abnormal lipid metabolism in the Chinese population: a cross-sectional study
}

\author{
Shaohong Zhang ${ }^{1,2 \#}$, Yingchai Zhang ${ }^{1,3 \#}$, Shuangjie Lin ${ }^{4}$, Shuguang Zhang ${ }^{3,5}$, Mingshan Qiu ${ }^{6,7}$ \\ ${ }^{1}$ Department of Endocrinology, Affiliated Hospital of Integrated Traditional Chinese and Western Medicine, Nanjing University of Chinese \\ Medicine, Nanjing, China; ${ }^{2}$ Department of Geriatrics, The Affiliated Huaian No.1 People's Hospital of Nanjing Medical University, Huai'an, \\ China; ${ }^{3}$ Nanjing University of Chinese Medicine, Nanjing, China; ${ }^{4}$ Department of TCM, Community Health Service Centre of the HeShan Street \\ in Xiamen city, Xiamen, China; ${ }^{5}$ Department of Cardiology, Huai'an Hospital of Traditional Chinese Medicine, Huai'an, China; ${ }^{6}$ Department of \\ Rheumatology, Xiamen Hospital, Beijing University of Chinese Medicine, Xiamen, China; ${ }^{7}$ Department of Rheumatology, Xiamen Hospital of \\ Traditional Chinese Medicine, Xiamen, China \\ Contributions: (I) Conception and design: Shaohong Zhang, Y Zhang; (II) Administrative support: M Qiu, Shuguang Zhang; (III) Provision of study \\ materials or patients: S Lin, Shaohong Zhang; (IV) Collection and assembly of data: S Lin; (V) Data analysis and interpretation: Y Zhang; (VI) \\ Manuscript writing: All authors; (VII) Final approval of manuscript: All authors. \\ "These authors contributed equally to this work. \\ Correspondence to: Shuguang Zhang. Huai'an Hospital of Traditional Chinese Medicine, Huai'an 223001, China. Email: fsyy02123@njucm.edu.cn; \\ Mingshan Qiu. Xiamen Hospital, Beijing University of Chinese Medicine, Xiamen 361000, China. Email: qiumingshan@sohu.com.
}

Background: Previous studies have reported that there may be a close relationship between elevated serum uric acid (UA) levels and metabolic syndrome. However, the association between these two factors has not been explored explicitly. Therefore, we carried out this study to investigate the association between UA and lipid profiles.

Methods: A total of 2,482 subjects participated in this cross-sectional study using a multistage stratified sampling method. Lipid profile, glucose metabolism, and other metabolic factors were measured and classified into UA-stratified and age-stratified groups to investigate the relationship between hyperuricemia and metabolic factors. Pearson correlations and logistic regressions were utilized to further explore the association between UA and lipid profile.

Results: The individuals aged 18 to 29 years presented with high serum UA concentrations. Moreover, the prevalence of hyperuricemia was higher among men than in women. Furthermore, statistically significant positive correlations were found between UA and serum triglycerides (TG), serum total cholesterol (TC), and low-density lipoprotein cholesterol (LDL-c). Conversely, only high-density lipoprotein cholesterol (HDL-c) was negatively correlated. Moreover, TG group status $(1.70 \leq \mathrm{TG}<2.30 \mathrm{mmol} / \mathrm{L})$ was an independent risk factor for hyperuricemia in both univariate and multivariate models.

Conclusions: This study found significant positive associations between TG, TC, LDL, and UA but an inverse relationship with HDL-c. Thus hyperuricemia may be a risk factor for abnormal lipid metabolism.

Keywords: Serum uric acid (UA); dyslipidemia; lipid profile; hyperuricemia; metabolic diseases

Submitted Aug 06, 2021. Accepted for publication Nov 09, 2021.

doi: 10.21037/apm-21-2734

View this article at: https://dx.doi.org/10.21037/apm-21-2734

^ ORCID: 0000-0001-5092-4859. 


\section{Introduction}

Purines are ubiquitous biomolecules that sustain life. Purines are generated by either or both of the two pathways: de novo purine biosynthesis or purine salvage (1). Serum uric acid (UA), the inert end-product of purine catabolism, is mainly produced by the liver and mostly excreted by the kidney $(2,3)$. The main causes of hyperuricemia are excessive production and/or decreased UA excretion. In China, the diagnostic criteria for hyperuricemia in both men and women is a serum UA $>420 \mu \mathrm{mol} / \mathrm{L}$ (4). A high-quality meta-analysis study, which includes articles published between 2000 and 2014, showed that the pooled prevalence of hyperuricemia in mainland China was $13.3 \%$ (95\% CI: $11.9-16.4 \%$ ) (5). According to the latest national cross-sectional study, the prevalence of hyperuricemia was $18.8 \%$ in people with adequate iodine status, while people living in iodine excessive areas are less likely to suffer hyperuricemia $13.7 \%$, which indicates that iodine status may have effects on uric acid levels (6). The total prevalence of hyperuricemia in the southwest of China was $20.08 \%$ in 2017, with the majority belonging to the 20-29 age group for both men and women (7). The high prevalence of hyperuricemia in this young age group is of concern.

The relationship between serum UA levels and lipid profiles has attracted considerable attention for some time. Hyperuricemia is widely considered a key risk factor for dyslipidemia, as some studies have shown a positive association between serum UA levels and lipid profiles in the adult populations of Bangladesh, Italy and the USA (8-10). Moreover, a high UA level is recognized as an independent risk factor for hypertriglyceridemia (11). However, a recent report does not show that serum UA was associated with metabolic syndrome, which is commonly characterized by dyslipidemia, hypertension, and type 2 diabetes (12). The relationship between serum UA levels and dyslipidemia has not been fully elucidated. Additionally, large-scale based studies on Chinese people to investigate the harm hyperuricemia are still to be confirmed. Therefore, we performed a cross-sectional study including 2482 adult participants and performing in both Jiangsu and Fujian Province to assess the relationship between serum UA and lipid profiles, glucose metabolism, other metabolites, and metabolic parameters in UAspecific and age-stratified groups to identify the risk factors for hyperuricemia. We present the following article in accordance with the STROBE reporting checklist (available at https://dx.doi.org/10.21037/apm-21-2734).

\section{Methods}

\section{Study participants and survey protocol}

Using a multistage, stratified sampling design, a total of 2,854 adults from both urban and rural areas of the Jiangsu and Fujian Provinces were selected and enrolled in this study. Adults who had lived in these areas for at least six months were included. The exclusion criteria were as follows: pregnancy; individuals who suffered from severe adrenal insufficiency, renal failure, or other severe comorbidities; and patients taking drugs that affect UA concentration and lipid metabolism. Finally, 2,482 subjects met our criteria and were included in the analysis. All procedures performed in this study involving human participants were in accordance with the Declaration of Helsinki (as revised in 2013). This study was approved by the Ethics Committee of the Affiliated Hospital of Integrated Traditional Chinese and Western Medicine, Nanjing University of Chinese Medicine (2021-LWKY007), and all participants provided written informed consent.

\section{Laboratory tests and physical examination}

Blood samples were collected after overnight fasting for at least $10 \mathrm{~h}$. Blood centrifugation was performed within $30 \mathrm{~min}$ after collection, and samples were stored at $-20{ }^{\circ} \mathrm{C}$. Fasting blood glucose (FBG), two-hour blood glucose, serum triglycerides (TG), total cholesterol (TC), high-density lipoprotein cholesterol (HDL-c), lowdensity lipoprotein cholesterol (LDL-c) and UA levels were measured using an automatic biochemical machine (COBAS 8000, Roche, Basel, Switzerland). Hemoglobin A1c (HbA1c) was measured using high-performance liquid chromatography (VARIANT II Hemoglobin Analyzer, BioRad, Hercules, CA, USA). Blood pressure was measured using an electronic blood pressure monitor (Omron HEM 7430, Omron Corporation, Kyoto, USA) twice after a fiveminute rest.

\section{Reference ranges and diagnosis criteria}

In the present study, hyperuricemia was defined as UA $>420 \mu \mathrm{mol} / \mathrm{L}$, following the Guidelines for the Diagnosis and Management of Hyperuricemia and Gout in China (4). UA levels were classified into four categories according to their interquartile ranges: Q1 $<243 \mu \mathrm{mol} / \mathrm{L}, 243 \leq \mathrm{Q} 2$ $<283 \mu \mathrm{mol} / \mathrm{L}, 283 \leq \mathrm{Q} 3<343 \mu \mathrm{mol} / \mathrm{L}$, and $\mathrm{Q} 4 \geq 343 \mu \mathrm{mol} / \mathrm{L}$. 
Lipid profile stratifications were classified in accordance with the Guidelines for Prevention and Treatment of Dyslipidemia in Adults in China (13). Body mass index (BMI) was categorized as underweight $\left(<18.5 \mathrm{~kg} / \mathrm{m}^{2}\right)$, normal weight $\left(18.5 \leq \mathrm{BMI}<24.0 \mathrm{~kg} / \mathrm{m}^{2}\right)$, overweight $\left(24.0 \leq\right.$ BMI $\left.<28.0 \mathrm{~kg} / \mathrm{m}^{2}\right)$, and obese $\left(\geq 28 \mathrm{~kg} / \mathrm{m}^{2}\right)(14)$. Chinese guidelines are available on blood pressure and serum glucose concentration categories $(15,16)$. Normal thyroid-stimulating reference values ranged from 0.27 to $4.20 \mathrm{mIU} / \mathrm{L}$, according to the manufacturer's instructions.

\section{Statistical analysis}

All statistical analyses were performed using SPSS software (version 26.0; SPSS, Inc., Chicago, IL, USA). Normally distributed data are presented as means (SD), while variables with skewed distributions are presented as medians (interquartile ranges). Differences among age groups or UA quartile groups were compared using one-way analysis of variance tests. Chi-square tests were performed to determine the distributions, percentages, and associations between UA and other factors. Pearson correlation analysis was performed to determine the association between UA and lipid profiles. Both univariate and multivariate logistic regression analyses were used to investigate the risk factors for hyperuricemia. For all measures, a two-tailed $\mathrm{P}$ value of $<0.05$ was considered as indicating statistical significance.

\section{Results}

\section{Characteristics of individuals in the study cobort}

A total of 2,482 participants (1,249 males and 1,233 females) were enrolled in the present study (Table 1). The majority of individuals were younger than 60 years $(84.5 \%$ men and $85.6 \%$ women). UA concentrations displayed an opposite tendency between the sexes, decreasing with age in males and increasing with age in females. According to the results above, the following analyses revealed that menopausal women were prone to have a higher UA concentration (Tables S1 and S2). The 18-29-year-old group had high levels of UA in both sexes. TC and LDL-c levels increased with aging but had a relatively lower level among women. Older participants had higher average blood pressure compared to younger participants. As for metabolic biomarkers, most participants had a normal weight and a normal range of FBG, two-hour postprandial blood glucose (2hPB), and HbA1c. Regarding thyroid-related values, TSH levels also showed an increasing trend with age in both sexes.

\section{Characteristics of individuals stratified by UA quartiles}

To investigate the association between UA and other metabolic biomarkers, we stratified individuals according to UA quartiles: Q1 (UA $<234 \mu \mathrm{mol} / \mathrm{L}), \mathrm{Q} 2(234 \leq \mathrm{UA}$ $<284 \mu \mathrm{mol} / \mathrm{L}), \mathrm{Q} 3(284 \leq \mathrm{UA}<345 \mu \mathrm{mol} / \mathrm{L})$, and Q4 (UA $\geq 345 \mu \mathrm{mol} / \mathrm{L}$ ). The average age of each group was slightly more than 40 years (Table 2). Similar to the age-stratified groups, we also found an inverse tendency for sex in this UA quartile stratification. The Q4 was composed mainly of males ( $42.2 \%$ males, $7.5 \%$ females). By contrast, the Q1 group had the largest proportion of females (43.3\%) (Table 2). Further investigations showed that women who were menopausal had higher serum UA levels compared with those who premenopausal (Table S2). Regarding the lipid profile, TG, TC, and LDL-c increased from Q1 to Q4. Notably, participants in the Q3 and Q4 groups were overweight, with an average BMI of 24.06 and $25.20 \mathrm{~kg} / \mathrm{m}^{2}$, respectively. However, the first two groups had a normal weight. No statistical differences were found for FBG, 2hPBG, and HbA1c levels.

\section{Prevalence of hyperuricemia in groups with different age stratifications}

The total prevalence of hyperuricemia was $5.9 \%$ in our study, with $10.7 \%$ and $1.1 \%$ for men and women, respectively. Stratified by age groups, the prevalence of hyperuricemia presented as a U-shaped curve with the nadir at the 60-69-year-old group for both sexes, with peaks appearing for the youngest group (16.7\% for men and $1.3 \%$ for women). Regardless of age group, the prevalence was consistently higher in men than in women (Figure 1).

\section{Association between $U A$ and lipid profile}

Spearman correlation analysis was performed to investigate the association between UA and lipid profiles (Figure 2). A statistically significant positive association was revealed between UA and TG, TC, and LDL-c $(r=0.275, r=0.117$, $\mathrm{r}=0.180$, respectively; all $\mathrm{P}<0.001$ ). By contrast, Figure $2 C$ shows that only HDL-c had a negative correlation with UA $(\mathrm{r}=-0.268, \mathrm{P}<0.001)$.

Logistic regression analyses were also performed to evaluate the key risk factors for hyperuricemia (Table 3). In 
Table 1 Characteristics of individuals across different age-stratified groups by sex

\begin{tabular}{|c|c|c|c|c|c|c|c|c|}
\hline Characteristics & Total & \multicolumn{6}{|c|}{ Age group (years) } & $P$ \\
\hline \multicolumn{9}{|l|}{ Male } \\
\hline UA & $330.37(67.58)$ & $348.96(66.73)$ & $339.32(65.70)$ & $328.63(65.83)$ & $315.59(69.11)$ & $310.46(63.60)$ & $310.46(64.31)$ & $<0.001$ \\
\hline $\mathrm{TG}$ & $1.57(1.29)$ & $1.25(0.85)$ & $1.80(1.40)$ & $1.94(1.85)$ & $1.54(0.83)$ & $1.35(0.96)$ & $1.06(0.50)$ & $<0.001$ \\
\hline HDL-c & $1.38(0.34)$ & $1.31(0.27)$ & $1.31(0.29)$ & $1.36(0.36)$ & $1.44(0.39)$ & $1.53(0.40)$ & $1.52(0.36)$ & $<0.001$ \\
\hline LDL-C & $2.60(0.71)$ & $2.24(0.63)$ & $2.63(0.68)$ & $2.69(0.69)$ & $2.74(0.75)$ & $2.86(0.66)$ & $2.80(0.72)$ & $<0.001$ \\
\hline SBP & $124[15]$ & 117 [12] & $120[12]$ & $125[14]$ & $129[17]$ & $132[18]$ & $134[16]$ & $<0.001$ \\
\hline DBP & $82[10]$ & $77[8]$ & $81[9]$ & $84[10]$ & $85[11]$ & $85[11]$ & 84 [10] & $<0.001$ \\
\hline $2 \mathrm{hPG}$ & $6.33(2.35)$ & $5.21(1.22)$ & $5.98(2.12)$ & $6.62(2.57)$ & $7.14(2.16)$ & $7.65(3.25)$ & $6.90(2.27)$ & $<0.001$ \\
\hline $\mathrm{HbA1c}$ & $5.33(0.74)$ & $5.05(0.36)$ & $5.25(0.77)$ & $5.36(0.64)$ & $5.51(0.84)$ & $5.63(0.92)$ & $5.47(1.02)$ & $<0.001$ \\
\hline TSH & $2.78(4.60)$ & $2.83(1.39)$ & $2.88(6.17)$ & $2.41(1.20)$ & 2.30 (1.39) & $2.47(1.64)$ & $5.06(12.64)$ & $<0.001$ \\
\hline \multicolumn{9}{|l|}{ Female } \\
\hline UA & $249.99(59.34)$ & $257.43(60.57)$ & $240.09(56.55)$ & $237.33(58.39)$ & 253.57 (54.39) & 264.35 (57.36) & $266.55(67.41)$ & $<0.001$ \\
\hline TG & $1.30(0.88)$ & $0.96(0.47)$ & $1.10(0.66)$ & $1.39(1.04)$ & $1.56(1.03)$ & $1.74(1.13)$ & $1.48(0.65)$ & $<0.001$ \\
\hline TC & $4.62(0.95)$ & $4.00(0.71)$ & $4.30(0.74)$ & $4.73(0.84)$ & $5.00(0.88)$ & $5.31(0.95)$ & $5.54(0.93)$ & $<0.001$ \\
\hline HDL-c & $1.52(0.36)$ & $1.52(0.32)$ & $1.49(0.31)$ & $1.51(0.36)$ & $1.53(0.38)$ & $1.52(0.45)$ & $1.67(0.45)$ & 0.002 \\
\hline $2 \mathrm{hPG}$ & $6.74(2.11)$ & $5.58(1.22)$ & $6.45(1.61)$ & $6.98(1.96)$ & $7.47(2.17)$ & $8.28(2.87)$ & 7.97 (2.94) & $<0.001$ \\
\hline $\mathrm{HbA1c}$ & $5.31(0.79)$ & $4.99(0.41)$ & $5.11(0.40)$ & $5.27(0.69)$ & $5.63(1.06)$ & $5.83(1.11)$ & $5.69(0.94)$ & $<0.001$ \\
\hline TSH & $3.20(4.71)$ & $3.19(5.76)$ & $2.86(2.35)$ & 3.08 (4.68) & $3.31(3.12)$ & $2.88(1.52)$ & 4.78 (9.39) & 0.037 \\
\hline
\end{tabular}

1. UA, TG, TC, HDL-C, LDL-C, SBP, DBP, BMI, FBG, 2hPB, HbA1c, and TSH are presented as means (SD). 2. Missing values: 1 for SBP, 1 for DBP, 4 for FBG, 179 for PBG, 21 for HbA1c. UA, serum uric acid; TG, serum triglyceride; TC, serum total cholesterol; HDL-C, highdensity lipoprotein cholesterol; LDL-C, low-density lipoprotein cholesterol; SBP, systolic blood pressure; DBP, diastolic blood pressure; BMI, body mass index; FBG, fasting blood glucose; 2hPB, two-hour postprandial blood glucose; HbA1c, hemoglobin A1c; TSH, thyroidstimulating hormone.

the univariate model, compared with TG $<1.70 \mathrm{mmol} / \mathrm{L}$, both $1.70 \leq \mathrm{TG}<2.30 \mathrm{mmol} / \mathrm{L}$ and $\mathrm{TG} \geq 2.30 \mathrm{mmol} / \mathrm{L}$ were risk factors for hyperuricemia [odds ratio $(\mathrm{OR})=2.279,95 \%$ confidence interval (CI): 1.463-3.549; OR $=3.09595 \%$ CI: 2.061-4.648, respectively]. The multivariate model further confirmed that $1.70 \leq \mathrm{TG}<2.30 \mathrm{mmol} / \mathrm{L}$ was an independent risk factor for hyperuricemia $(\mathrm{OR}=2.028,95 \%$ CI: 1.250-3.291). In comparison with the individuals in the $\mathrm{TC}<5.20 \mathrm{mmol} / \mathrm{L}$ group, those in the group with TC $\geq 6.20 \mathrm{mmol} / \mathrm{L}$ were more likely to suffer from hyperuricemia 
Table 2 Characteristics of different uric-acid-stratified groups

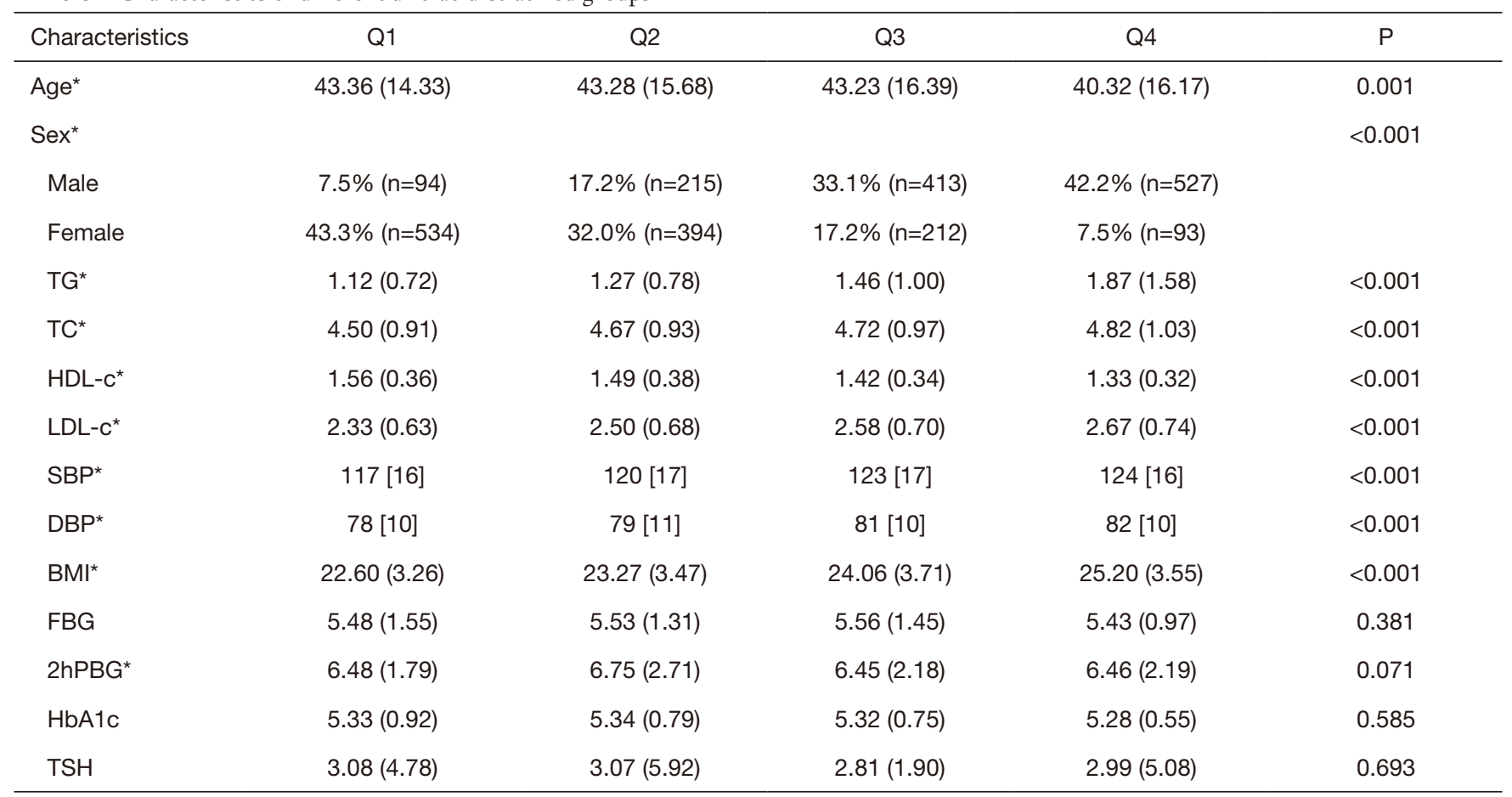

1. Q1 serum uric acid (UA) <234 $\mu \mathrm{mol} / \mathrm{L} ; \mathrm{Q} 2234 \leq \mathrm{UA}<283 \mu \mathrm{mol} / \mathrm{L} ; \mathrm{Q} 3283 \leq \mathrm{UA}<343 \mu \mathrm{mol} / \mathrm{L} ; \mathrm{Q} 4 \mathrm{UA} \geq 343 \mu \mathrm{mol} / \mathrm{L} .2 .{ }^{*}$, P<0.05. 3. Age, TG, TC, HDL-C, LDL-C, TSH, SBP, DBP, BMI, FBG, 2hPB, HbA1c are presented as means (SD). Sex is presented as percentage (counts). 4. Missing values: 1 for SBP, 1 for DBP, 4 for FBG, 179 for PBG, 21 for HbA1c. TG, serum triglyceride; TC, serum total cholesterol; HDL-C, high-density lipoprotein cholesterol; LDL-C, low-density lipoprotein cholesterol; TSH, thyroid-stimulating hormone; SBP, systolic blood pressure; DBP, diastolic blood pressure; BMI, body mass index; FBG, fasting blood glucose; 2hPB, two-hour postprandial blood glucose; $\mathrm{HbA1c}$, hemoglobin A1c.

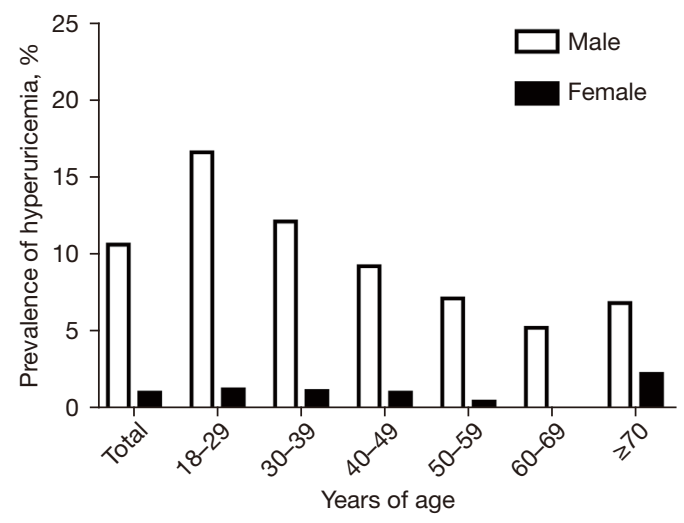

Figure 1 Prevalence of hyperuricemia by age-stratified groups.

$(\mathrm{OR}=7.746,95 \% \mathrm{CI}: 2.323-17.427)$. With LDL-c $<2.60 \mathrm{mmol} / \mathrm{L}$ as reference group, both $2.60 \leq \mathrm{LDL}-\mathrm{c}$ $<3.40 \mathrm{mmol} / \mathrm{L}$ and LDL-c $\geq 4.10 \mathrm{mmol} / \mathrm{L}$ were risk factors for hyperuricemia (OR $=2.846,95 \%$ CI: $1.628-4.975$; OR $=5.029,95 \%$ CI: $2.707-9.341)$. By contrast, participants who had a higher level of HDL-c (HDL-c $>1.0 \mathrm{mmol} / \mathrm{L}$ ) seemed to be affected less by hyperuricemia $(\mathrm{OR}=0.405$, 95\% CI: 0.257-0.640). Additionally, female sex was also a protective factor against hyperuricemia $(\mathrm{OR}=0.089,95 \% \mathrm{CI}$ : $0.050-0.158$ ), while people who were obese were at increased risk for hyperuricemia (OR $=2.914,95 \% \mathrm{CI}: 1.874-4.530$, adjusted OR $=1.974,95 \%$ CI: 1.183-3.293).

\section{Discussion}

Compared with the other age groups, our results showed that individuals aged 18 to 29 years presented with high serum UA concentrations, which may be related to their living standards and lifestyles. First, the improvements in living standards mean that there is no shortage of food supply. Indeed, individuals in this younger age group may have excessive food intake, which significantly increases the risk of hyperuricemia. Young people with heavy study loads also typically have a sedentary lifestyle and may thus be 

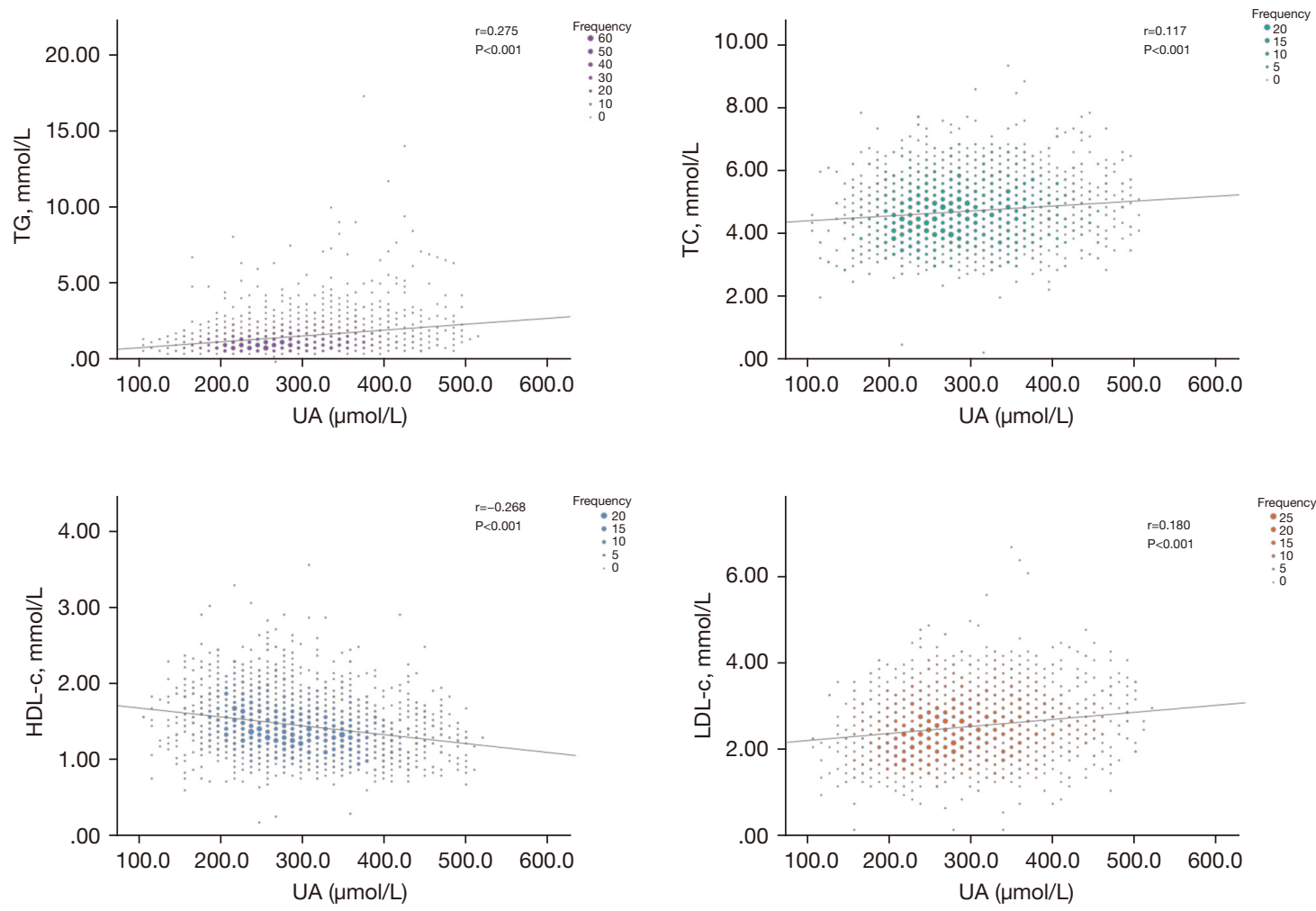

Figure 2 Associations between serum uric acid and serum triglycerides, serum total cholesterol, and high- and low-density lipoprotein cholesterol.

compromising their physical health to excel academically. Several studies have reported that unhealthy lifestyles, such as staying up late, excessive amounts of alcohol, and a sedentary lifestyle, are all risk factors for hyperuricemia $(17,18)$. The two main risk factors of excessive food intake and unhealthy lifestyle, might explain why participants aged 18-29 years presented with a higher UA concentration than participants in the other age groups.

Additionally, we found that in all the age groups, the prevalence of hyperuricemia was higher among men than in women (Figure 1). Moreover, we also found that the level of serum UA increased with age in women, whereas the opposite trend was observed in men. A similar result has been reported in southwestern China (7). Investigating the association between sex and age, our results show that menopausal women showed a higher level of serum UA compared with premenopausal women. Several factors contribute to these differences (Tables S1 and S2). First, men consume higher levels of purine and alcohol as part of their diet, which are risk factors for hyperuricemia $(19,20)$. Second, sex hormones may partly explain these differences.
Serum UA levels have been reported to be negatively correlated with testosterone levels (21). Testosterone affects the expression pattern of urate transporters in the kidneys, such as urate transporter 1, glucose transporter 9 , and sodium-coupled monocarboxylate transporter 1 , which induces UA reabsorption in males (22). In the liver, testosterone increases the activity of the rate-limiting enzyme xanthine oxidase, which generates UA (7). In females, the lower levels of serum UA may be related to the effect of estrogen on postsecretory tubular reabsorption of UA (23). Hence, we hypothesized that the prevalence of hyperuricemia in men would be higher than that in women. Moreover, estrogen levels decrease following menopause, which may explain why the prevalence of hyperuricemia increases in postmenopausal women.

The present study also found a significant positive relationship between serum UA levels and TG, TC, LDL-c, and BMI and an inverse relationship between serum UA and HDL-c, which was also observed in previous studies $(8,10)$. Furthermore, we found that $1.70 \leq \mathrm{TG}<2.30 \mathrm{mmol} / \mathrm{L}$ and a $\mathrm{BMI} \geq 28.0 \mathrm{~kg} / \mathrm{m}^{2}$ (obesity) were independent risk 
Table 3 Risk factors for hyperuricemia

\begin{tabular}{|c|c|c|c|c|}
\hline Variable & \multicolumn{2}{|c|}{ Univariate } & \multicolumn{2}{|c|}{ Multivariate } \\
\hline Female & $0.089(0.050,0.158)$ & $<0.001$ & $0.105(0.059,0.189)$ & $<0.001$ \\
\hline \multicolumn{5}{|l|}{ Age } \\
\hline 30-39-year group & 1.000 (reference) & - & 1.000 (reference) & \\
\hline 40-49-year group & $0.750(0.453,1.243)$ & 0.264 & $0.713(0.418,1.217)$ & 0.215 \\
\hline 50-59-year group & $0.542(0.292,1.004)$ & 0.051 & $0.595(0.310,1.144)$ & 0.119 \\
\hline 60-69-year group & $0.400(0.176,0.913)$ & 0.029 & $0.433(0.182,1.031)$ & 0.059 \\
\hline$\geq 70$-year group & $0.660(0.301,1.448)$ & 0.300 & $0.875(0.377,2.029)$ & 0.755 \\
\hline $\mathrm{TG} \geq 2.30 \mathrm{mmol} / \mathrm{L}$ & $3.095(2.061,4.648)$ & $<0.001$ & $1.327(0.538,3.273)$ & 0.539 \\
\hline \multicolumn{5}{|l|}{$\mathrm{TC}$} \\
\hline $\mathrm{TC}<5.20 \mathrm{mmol} / \mathrm{L}$ & 1.000 (reference) & - & 1.000 (reference) & - \\
\hline $5.20 \leq \mathrm{TC}<6.20 \mathrm{mmol} / \mathrm{L}$ & $3.351(0.389,28.878)$ & 0.271 & $1.529(0.127,18.409)$ & 0.738 \\
\hline $\mathrm{TC} \geq 6.20 \mathrm{mmol} / \mathrm{L}$ & $7.746(2.323,17.427)$ & $<0.001$ & $3.197(0.811,12.613)$ & 0.097 \\
\hline \multicolumn{5}{|l|}{ HDL-c } \\
\hline $\mathrm{HDL}-\mathrm{c}<1.0 \mathrm{mmol} / \mathrm{L}$ & 1.000 (reference) & - & 1.000 (reference) & - \\
\hline LDL-c $\geq 4.10 \mathrm{mmol} / \mathrm{L}$ & $5.029(2.707,9.341)$ & $<0.001$ & $1.935(0.509,7.352)$ & 0.332 \\
\hline \multicolumn{5}{|l|}{ SBP } \\
\hline $\mathrm{SBP}<140 \mathrm{mmHg}$ & 1.000 (reference) & - & 1.000 (reference) & - \\
\hline $\mathrm{SBP} \geq 140 \mathrm{mmHg}$ & $1.145(0.756,1.733)$ & 0.522 & $1.145(0.644,2.034)$ & 0.644 \\
\hline \multicolumn{5}{|l|}{ DBP } \\
\hline $\mathrm{DBP}<90 \mathrm{mmHg}$ & 1.000 (reference) & - & 1.000 (reference) & - \\
\hline $\mathrm{DBP} \geq 90 \mathrm{mmHg}$ & $1.285(0.188,1.285)$ & 0.188 & $0.908(0.548,1.505)$ & 0.708 \\
\hline \multicolumn{5}{|l|}{ BMI } \\
\hline $18.5 \leq \mathrm{BMI}<24.0 \mathrm{~kg} / \mathrm{m}^{2}$ & 1.000 (reference) & & 1.000 (reference) & - \\
\hline $\mathrm{BMI}<18.5 \mathrm{~kg} / \mathrm{m}^{2}$ & $0.274(0.066,1.135)$ & 0.074 & $0.212(0.050,0.900)$ & 0.035 \\
\hline $24.0 \leq \mathrm{BMl}<27.9 \mathrm{~kg} / \mathrm{m}^{2}$ & $1.628(1.101,2.406)$ & 0.015 & $1.374(0.889,2.124)$ & 0.153 \\
\hline $\mathrm{BMI} \geq 28.0 \mathrm{~kg} / \mathrm{m}^{2}$ & $2.914(1.874,4.530)$ & $<0.001$ & $1.974(1.183,3.293)$ & 0.009 \\
\hline
\end{tabular}

TG, triglyceride; TC, total cholesterol; HDL-c, lipoprotein cholesterol; LDL-c, low-density lipoprotein cholesterol; SBP, systolic blood pressure; DBP, diastolic blood pressure; BMI, body mass index. 
factors for hyperuricemia, while a BMI $<18.5 \mathrm{~kg} / \mathrm{m}^{2}$ was an independent protective factor. It is indisputable that obesity is an important component of metabolic syndrome. In particular, it is known that accumulation of visceral adipose tissue may be detrimental to UA metabolism. Specifically, the accumulation of visceral fat induces a large influx of plasma-free fatty acids into the liver and hepatic portal vein, which stimulates the synthesis of TG followed by an associated surge in the production of UA via activation of the UA synthesis pathway (24). However, both obesity and dyslipidemia are associated with a high risk of hyperuricemia, and elevated UA levels are also associated with an increased risk of obesity and dyslipidemia. Experimentally, UA has been shown to increase TG accumulation in cultured liver cells $(25,26)$. Apart from this, a restricted lifestyle does benefits on preventing hyperuricemia, which can be elucidated as restraining the intake of alcohol, high-purine food and abundant fructose. On the contrary diary and fresh vegetables are high recommended. Hyperuricemia increases the level of TG in the liver tissue of rats (27). This mechanism may be mediated by intracellular and mitochondrial oxidative stress. UA induces the activation of nicotinamide adenine dinucleotide phosphate oxidase preceding endoplasmic reticulum stress, which further induces the mitochondrial production of reactive oxygen species in hepatocytes (25). Furthermore, allopurinol treatment reduces the levels of TG, TC, and fat lipid accumulation in rats (28). Considering all of these factors, the interactions between lipid and uric metabolism should be considered in the clinical treatment of metabolic diseases.

Hyperuricemia is commonly found in patients with obesity, metabolic syndrome, and type 2 diabetes. However, we found no association between serum UA levels and FBG, 2hPG, and HbA1c levels. Hyperuricemia was previously considered a secondary consequence of insulin resistance. However, recent studies have shown that it may have a certain causal role, especially given that elevated serum UA levels often precede the development of insulin resistance (29-31). It is well known that thyroid hormones affect the synthesis, mobilization, and degradation of lipids. As such, thyroid dysfunction (especially overt or subclinical hypothyroidism) has a negative effect on lipid metabolism, leading to hypercholesterolemia, which in turn results in an increased risk of cardiovascular diseases (32). It has been reported that thyroid hormones can modulate UA metabolism in patients with late-onset subclinical hypothyroidism. In normal thyroid function, UA levels have a linear correlation with FT3 and FT4 (33). However, in this cross-sectional study, we found no association between serum UA and TSH levels.

This study had several limitations. First, it is a crosssectional study that was undertaken in the Jiangsu and Fujian Provinces of China, and as such may reflect local conditions. We recommend that further studies be carried out to confirm our results. Our sample size was limited. Thus, future studies with larger samples are needed to confirm our results. Lastly, we did not measure lipid and purine levels of participants' diets, and the rigor of the study would have been enhanced had such measurements been included.

In conclusion, the present study demonstrated a significant association between serum UA levels and lipid profiles in inhabitants of the Jiangsu and Fujian Provinces of China. Further studies are required to identify the underlying mechanisms.

\section{Acknowledgments}

Funding: This study was funded by the Practice Innovation Program of Jiangsu Province (KYCX19_1184) and Huai'an Science and Technology Plan (Natural Science Research Program, HABZ201813).

\section{Footnote}

Reporting Checklist: The authors have completed the STROBE reporting checklist. Available at https://dx.doi. org/10.21037/apm-21-2734

Data Sharing Statement: Available at https://dx.doi. org/10.21037/apm-21-2734

Conflicts of Interest: All authors have completed the ICMJE uniform disclosure form (available at https://dx.doi. org/10.21037/apm-21-2734). All authors report that this study was funded by the Practice Innovation Program of Jiangsu Province (KYCX19_1184) and Huai'an Science and Technology Plan (Natural Science Research Program, HABZ201813). The authors have no other conflicts of interest to declare.

Ethical Statement: The authors are accountable for all aspects of the work in ensuring that questions related to the accuracy or integrity of any part of the work are appropriately investigated and resolved. All procedures 
performed in this study involving human participants were in accordance with the Declaration of Helsinki (as revised in 2013). This study was approved by the Ethics Committee of the Affiliated Hospital of Integrated Traditional Chinese and Western Medicine, Nanjing University of Chinese Medicine (2021-LWKY-007), and all participants provided written informed consent.

Open Access Statement: This is an Open Access article distributed in accordance with the Creative Commons Attribution-NonCommercial-NoDerivs 4.0 International License (CC BY-NC-ND 4.0), which permits the noncommercial replication and distribution of the article with the strict proviso that no changes or edits are made and the original work is properly cited (including links to both the formal publication through the relevant DOI and the license). See: https://creativecommons.org/licenses/by-nc-nd/4.0/.

\section{References}

1. Pareek V, Pedley AM, Benkovic SJ. Human de novo purine biosynthesis. Crit Rev Biochem Mol Biol 2021;56:1-16.

2. Kanbay M, Jensen T, Solak Y, et al. Uric acid in metabolic syndrome: From an innocent bystander to a central player. Eur J Intern Med 2016;29:3-8.

3. Fiorentino TV, Sesti F, Succurro E, et al. Higher serum levels of uric acid are associated with a reduced insulin clearance in non-diabetic individuals. Acta Diabetol 2018;55:835-42.

4. Chinese Society of Endocrinology. Guidelines for the Diagnosis and Management of Hyperuricemia and Gout in China (2019). Chin J Endocrinol Metab 2020;2020:1-13.

5. Liu R, Han C, Wu D, et al. Prevalence of Hyperuricemia and Gout in Mainland China from 2000 to 2014: A Systematic Review and Meta-Analysis. Biomed Res Int 2015;2015:762820.

6. Lu X, Shi X, Li Y, et al. A negative association between urinary iodine concentration and the prevalence of hyperuricemia and gout: a cross-sectional and population-based study in Mainland China. Eur J Nutr 2020;59:3659-68.

7. Liu DM, Jiang LD, Gan L, et al. Association between serum uric acid level and body mass index in sex- and age-specific groups in southwestern China. Endocr Pract 2019;25:438-45.

8. Ali N, Rahman S, Islam S, et al. The relationship between serum uric acid and lipid profile in Bangladeshi adults.
BMC Cardiovasc Disord 2019;19:42.

9. Lippi G, Montagnana M, Luca Salvagno G, et al. Epidemiological association between uric acid concentration in plasma, lipoprotein(a), and the traditional lipid profile. Clin Cardiol 2010;33:E76-80.

10. Peng TC, Wang CC, Kao TW, et al. Relationship between hyperuricemia and lipid profiles in US adults. Biomed Res Int 2015;2015:127596.

11. Keenan T, Blaha MJ, Nasir K, et al. Relation of uric acid to serum levels of high-sensitivity C-reactive protein, triglycerides, and high-density lipoprotein cholesterol and to hepatic steatosis. Am J Cardiol 2012;110:1787-92.

12. Li L, Song Q, Yang X. Lack of associations between elevated serum uric acid and components of metabolic syndrome such as hypertension, dyslipidemia, and T2DM in Overweight and Obese Chinese Adults. J Diabetes Res 2019;2019:3175418.

13. China Joint Committee for the Revision of Adult Dyslipidemia Guidelines. Guidelines for prevention and treatment of dyslipidemia in adults in China. Chin Circul J 2016;31:937-50.

14. Obesity Group of Chinese Society of Endocrinology. Consensus on prevention and treatment of adult obesity in China. Chin J Endocrinol Metab 2011;27:711-7.

15. Joint Committee for Guideline Revision. 2018 Chinese Guidelines for Prevention and Treatment of Hypertension-A report of the Revision Committee of Chinese Guidelines for Prevention and Treatment of Hypertension. J Geriatr Cardiol 2019;16:182-241.

16. Jia W, Weng J, Zhu D, et al. Standards of medical care for type 2 diabetes in China 2019. Diabetes Metab Res Rev 2019;35:e3158.

17. Park DY, Kim YS, Ryu SH, et al. The association between sedentary behavior, physical activity and hyperuricemia. Vasc Health Risk Manag 2019;15:291-9.

18. Choi HK. A prescription for lifestyle change in patients with hyperuricemia and gout. Curr Opin Rheumatol 2010;22:165-72.

19. Choi HK, Liu S, Curhan G. Intake of purine-rich foods, protein, and dairy products and relationship to serum levels of uric acid: the Third National Health and Nutrition Examination Survey. Arthritis Rheum 2005;52:283-9.

20. Li Y, Jiang Y, Zhang M, et al. Drinking behaviour among men and women in China: the 2007 China Chronic Disease and Risk Factor Surveillance. Addiction 2011;106:1946-56.

21. Han Y, Zhang Y, Cao Y, et al. Exploration of the association between serum uric acid and testosterone in 
adult males: NHANES 2011-2016. Transl Androl Urol 2021;10:272-82.

22. Kanda E, Muneyuki T, Kanno Y, et al. Uric acid level has a U-shaped association with loss of kidney function in healthy people: a prospective cohort study. PLoS One 2015; 10:e0118031.

23. Jung JH, Song GG, Lee YH, et al. Serum uric acid levels and hormone therapy type: a retrospective cohort study of postmenopausal women. Menopause 2018;25:77-81.

24. Ali N, Perveen R, Rahman S, et al. Prevalence of hyperuricemia and the relationship between serum uric acid and obesity: A study on Bangladeshi adults. PLoS One 2018;13:e0206850.

25. Lanaspa MA, Sanchez-Lozada LG, Choi YJ, et al. Uric acid induces hepatic steatosis by generation of mitochondrial oxidative stress: potential role in fructosedependent and -independent fatty liver. J Biol Chem 2012;287:40732-44.

26. Choi YJ, Shin HS, Choi HS, et al. Uric acid induces fat accumulation via generation of endoplasmic reticulum stress and SREBP-1c activation in hepatocytes. Lab Invest 2014;94:1114-25.

27. Tapia E, Cristóbal M, García-Arroyo FE, et al. Synergistic effect of uricase blockade plus physiological amounts of fructose-glucose on glomerular hypertension and oxidative stress in rats. Am J Physiol Renal Physiol 2013;304:F727-36.

28. Wang W, Wang C, Ding XQ, et al. Quercetin and allopurinol reduce liver thioredoxin-interacting protein to alleviate inflammation and lipid accumulation in diabetic rats. Br J Pharmacol 2013;169:1352-71.

29. King C, Lanaspa MA, Jensen T, et al. Uric Acid as a Cause of the Metabolic Syndrome. Contrib Nephrol 2018;192:88-102.

30. Krishnan E, Pandya BJ, Chung L, et al. Hyperuricemia in young adults and risk of insulin resistance, prediabetes, and diabetes: a 15-year follow-up study. Am J Epidemiol 2012;176:108-16.

31. Johnson RJ, Nakagawa T, Sanchez-Lozada LG, et al. Sugar, uric acid, and the etiology of diabetes and obesity. Diabetes 2013;62:3307-15.

32. Duntas LH, Brenta G. A Renewed Focus on the Association Between Thyroid Hormones and Lipid Metabolism. Front Endocrinol (Lausanne) 2018;9:511.

33. Chao G, Zhu Y, Fang L. Retrospective Analysis of the Correlation between Uric Acid and Thyroid Hormone in People with Normal Thyroid Function. J Diabetes Res 2019;2019:5904264.

(English Language Editor: B. Meiser)

Cite this article as: Zhang S, Zhang Y, Lin S, Zhang S, Qiu M. Hyperuricemia as a possible risk factor for abnormal lipid metabolism in the Chinese population: a cross-sectional study. Ann Palliat Med 2021;10(11):11454-11463. doi: 10.21037/apm-212734 
Supplementary

Table S1 Serum uric acid concentration by age-stratified groups in females

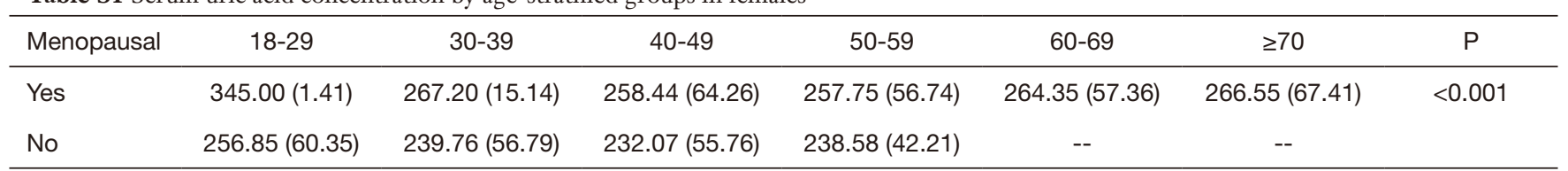

Some young participants were rendered menopausal due to medicine, surgery or other reasons.

Table S2 Serum uric acid concentrations in uric-acid-stratified groups in females

\begin{tabular}{lccccc}
\hline Menopausal & Q1 & Q2 & Q3 & Q4 & P \\
\hline Yes & $35.3 \%(146)$ & $29.7 \%(123)$ & $24.9 \%(103)$ & $10.1 \%(42)$ & $<0.001$ \\
No & $47.4 \%(388)$ & $33.1 \%(271)$ & $13.3 \%(109)$ & $6.2 \%(51)$ \\
\hline
\end{tabular}

